\title{
HARALD BOHR
}

\author{
April 22, 1887-January 22, 1951
}

Born in Copenhagen, Denmark, lived, learned and taught there, and died there; but liked to visit near and far. His father was Christian Bohr, Professor of physiology at the medical school; his mother was Jewish, and he had to flee the country in 1943, going then to Sweden for two years. During his last years he was Master of the one resident college in Copenhagen and he greatly enjoyed being surrounded by young people. Bohr was a very friendly person, kind and good-natured, chatty but not indiscreet, and he made and kept to his friends all his life. For all that, he was a shade melancholy, but this side of him was brightened by being married exceedingly happily. In his younger years he had been a football (or rather soccer) player of some renown, a rare enough occurrence among budding savants on the Continent. In after-years he would trounce a ball if he saw one, but he did not look much of an athlete.

Bohr's fame is his almost periodic functions. He did the theory in his mid-thirties, and very little in other topics afterwards. He wrote afterwards many papers in his theory, though, and rather substantial ones too. But the vim of the first papers did not return, and by now Bohr lives forth as much in the work of others as in his own. Privately, he gloried in the interest his theory has evoked and he would help to propagate it. He loved to lecture, and he lectured extremely well. In an individual lecture, no matter how learned the audience, he would first recite the definition and basic properties and make introductory comments; and he would do the same in print even when full references were included.

Whether he had an opinion as to future potentialities of the theory we do not know. Right now it is rather quiet about the theory, except for some distant rumbling in the field of automorphic functions, especially of several variables, which might precipitate in a new shower of almost periodicity. However that may be, it may be said that Harald Bohr has had the good fortune of having a very distinctive theory of magnitude very clearly and originally attached to his name, and this, after all, is as much as a mathematician may expect to secure for himself in a lifetime.

When Bohr was a student the study of mathematics at Copenhagen was not organized, and Bohr learned in a somewhat haphazard way from persons like the geometer Zeuthen, the astronomer Thiele, and the analyst Niels Nielsen (cylinder functions) who made a fierce 
play for mathematical rigor but not always very successfully; but he could not learn from J. L. W. V. Jensen, probably the greatest of them all, because he was an electrical engineer by profession and a self-taught mathematician, and had no connection with academic life. The course fare was generously supplemented by reading in books such as the second edition of C. Jordan's Cours d'analyse (of Jordan theorem fame) and the Zahlentheorie by Dirichlet and Dedekind.

Even before earning his masters degree in 1909 (the $\mathrm{Ph} . \mathrm{D}$. degree, which was quite a serious affair, followed in 1910) Bohr was led to the study of the theory of summability, mainly of Dirichlet series, and he dwelt on this topic quite a number of years. Summability, even in its pre-Tauberian era, used to be an important topic of study which even algebraists of the stature of Frobenius and Schur would dignify with their attention, and the reasons for this were manifold. First of all, to the uninitiated it exuded a certain mystique of esoterically conjuring up a limit where none had been before. In the second place (and this led to the most far-reaching consequences), Fejér had succeeded in "summing" the Fourier series of a continuous function towards its value, where DuBois Reymond had distressingly shown that the partial sums themselves need not so converge. And finally, and this must have been decisive for Bohr for the time being, it held out some hope of "moving-in" on the zeta function in its critical strip (or at least on $L$-series without a pole) where other means of intrusion had not been successful. This purpose proper was not to be attained, really, but a new view on summability did remain. Actually, Bohr's contribution was eventually absorbed in M. Riesz' more felicitous approach, and the resulting summability is named after Riesz, and not Bohr; but Bohr himself described his share in the theory in his article written jointly with $\mathrm{H}$. Cramér, Die neuere Entwicklung der analytischen Zahlentheorie in Encyklopädie der Math. Wiss. Bd. II-3, Heft. 6, pp. 722-849.

We might mention that this early work of his brought Bohr soon in contact with E. Landau, and he spent 1909-1910 in Göttingen, which place he used to revisit frequently. He also became a very close friend of Hardy's, and also knew Littlewood very well, and stayed at Oxford and Cambridge in 1913-1914; and finally he went for several months in 1914 to Lebesgue in Paris to study his measure from him first-hand.

We think that the dominant interest of Harald Bohr was diophantine approximation and (equi-) distribution of numbers, but in a certain broad sense that would not lead him to a study of Weyl sums 
and the Waring problem, say. Somehow, to Bohr, the entire theory of almost periodic functions was an emanation from these sources, and this reflects itself best in his own proof for his "completeness theorem" which is so unlike any given afterwards and, in a nontechnical sense, is the most arithmetical among them all. Bohr was also infatuated with the classical theorem of Kronecker itself, and he repeatedly would give a new proof for it, which after all was not such an urgent task when compared with all the new problems suggesting themselves. Also, several years before arriving at the new theory, and repeatedly afterward, Bohr studied the zeta function and related functions as regards average frequency with which values are attained in the critical strip.

Bohr was the creator of the almost periodic functions, and he gave them such an appropriate name that no need arose for turning them into "fonctions bohriennes." Actually Bohr had a forerunner in the person of P. Bohl, who came within sight of the theory but missed it, although he has to his credit at least one nontrivial theorem which Bohr could take over virtually without change. One might even claim that Lagrange had anticipated a part of the theory by his study of mean motion. But after all this is conceded, the fact remains that almost periodic functions were Bohr's in the same sense as Lie groups were Lie's and in a stronger and much more significant sense than Banach spaces were Banach's, say. Bohr's high points of achievement consist of his having broken through towards a general definition for his functions such as he has found it, and of having then succeeded in proving his completeness theorem in his own fussy manner, such as he did; and as regards the genesis of his definition it is worth looking up a little known paper of his in which a tentative version is sought after (Über eine quasi-periodische Eigenschaft Dirichletschen Reihen mit Anwendung auf die Dirichletschen L-funktionen, Math. Ann. vol. 85, pp. 115-122). Bohr's personal triumph in attaining these achievements must be assessed against the fact that Bohr had been an arithmetico-analyst only, and always remained one only, and that nevertheless the true orientation of his achievements was towards topology, general group theory, homogeneous spaces, and such like directions, all of which had been rather alien to most arithmeticoanalysts of his generation. We might perhaps point out for what it is worth that Hardy and Landau, both very prolific writers and close friends to Bohr, never took up the new topic themselves, or as much as published a note about it.

The brisk development of almost periodic functions on groups, as initiated by von Neumann, has by the very rapidity of its progress 
very quickly led to the sobering realization that after inherent compactifications have been introduced, the general theory is by actual scope, though not by initial intent, not larger than a theory of functions on compact groups only, or compact homogeneous spaces at best; and theories of functions on genuinely open groups are yet to be developed. In Euclidean space the current body of Fourier analysis including the theory of generalized Fourier integrals (alias "distributions temperées") is a partial answer to the problem, perhaps. But on open semi-simple group spaces, say, the developments are not yet foreseeable, unless the recent work of Gelfand and Neumark and others is an indication of them.

If we contemplate Bohr's work the thought suggests itself that some of what is best in algebra, group theory, and topology has been coming forth from the fullness and fertility of analysis and has been stimulated if not always brought to surface by analysts themselves; and that perhaps analysis is the stuff of which our mathematics is made of, after all.

SALOMON BochNeR 